\title{
Resolving Cross Domain Firewall Policy Anomalies
}

\author{
Abdul Raziya Sultana \\ Department of Computer Science and Engineering \\ Gudlavalleru Engineering College \\ Gudlavalleru, A.P, India
}

\author{
Amrutasagar Kavarthapu \\ Department of Computer Science and Engineering \\ Gudlavalleru Engineering College \\ Gudlavalleru, A.P, India
}

\begin{abstract}
For last few years, Firewall usage with regard to protected network emails is important. Its ability to process inbound and confident bundle moves and accept or eliminate those bundle in accordance with the processing is what keeps this systems and networks protected. But considering a vast organization's protection needs with regard to firewall program guidelines, typical home solutions won't be sufficient because such organizations are compounds of different subnets which require comfort during plan quality. The key technical restriction is that firewall program guidelines cannot be shared across different websites for easier control because a firewall program plan might include important info and this might be a potential protection cycle hole that can be utilized by harmful users. Although largest rule calculations methods help to some extent they are unsuccessful of performance in accordance with the presence of NAT (Network Address Translation) device across different subnets (domains). So here recommend improving the current solution with a quality decision tree classifier criteria applied at routers for achieving the comfort maintained firewall program plan abnormality solutions with regard to repetitive rules control. So it involves wireless router extension recommend to imitate this event and confirm conclusion in accordance with the results.
\end{abstract}

\section{Keywords}

Firewall Optimization. Privacy, Cross Domain, Access control rule policies.

\section{INTRODUCTION}

FIREWALLS are crucial in obtaining personal systems of businesses, organizations, and home systems. A firewall program is often placed at the entry between a personal system and the external system so that it can check each inbound or confident bundle and decide whether to agree to or eliminate the bundle based on its plan. A firewall program plan is usually specified as a sequence of guidelines, called Access Control List (ACL), and each concept has a predicate over several bundle headlines areas (i.e., resource IP, location IP, resource slot, location slot, and method type) and a choice (i.e., agree to and discard) for the packages that match the predicate. [2] The guidelines in a firewall program plan generally follow the first-match semantics, where the choice for a bundle is the decision of the first concept that the bundle suits in the plan. Each physical interface of a router/firewall is designed with two ACLs: one for filtration confident packages and the other one for filtration inbound packages [1]

Prior perform on firewall program marketing concentrates on either [13] intra firewall program marketing or [14] inter firewall program marketing within one management sector where the comfort of firewall program guidelines is not a issue. Intra-firewall marketing means improving only one firewall program. It is obtained by either eliminating repetitive guidelines or spinning guidelines. To best information, no past techniques perform concentrates on cross-domain privacy-preserving inter firewall marketing. This paper represents the first step in discovering this unidentified space. Here specifically concentrate on eliminating inter firewall plan redundancies in a privacypreserving way.

Different representations of ideas may be learned from a set of marked information such as, sensory systems, guidelines, and choice plants. Decision shrub studying is reasonably fast and precise. Here to studying on large information places is to parallelize the process of studying by utilizing choice plants. It is uncomplicated to reduce a decision shrub to guidelines and the final reflection used in this research includes a concept platform created from decision. The technique followed here is to break a huge information set into ' $n$ ' categories, then learn a choice shrub on each of the ' $n$ ' categories in similar. A choice shrub will be expanded on each of $n$ processor chips individually. After growing the $n$ decision plants, they must be mixed in some way. The choice plants stay individual trees and new illustrations are run through all or a part of the plants with a category choice made based on some meta-rules for mixing the results of individual shrub classifiers. Here objective is to have only one choice system after learning is done individually on the $\mathrm{n}$ subsets of information. The independent students can be considered as providers studying a little about a sector with the information of each broker to be mixed into one understanding. Towards this end the separate choice plants might be mixed into a single choice shrub.

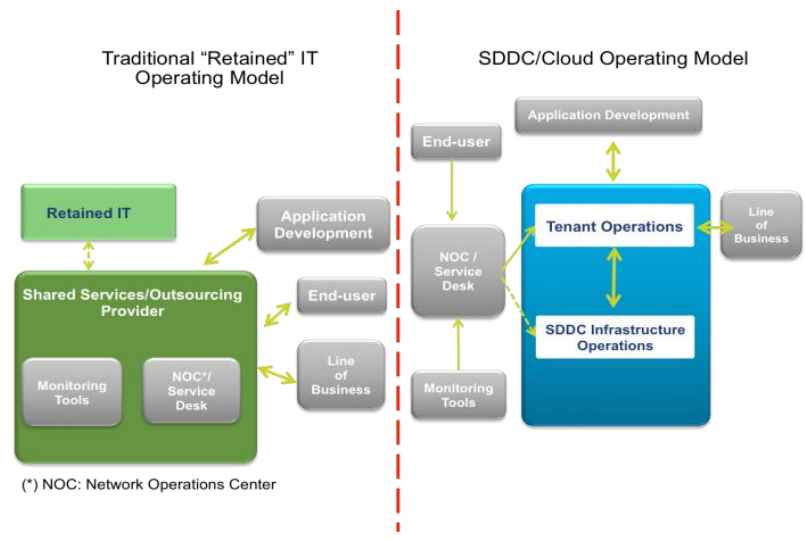

Fig 1: Cross-domain architecture with processing of cloud

\section{RELATED WORK}

At each node in a choice shrub a feature must be chosen to divide the node's illustrations into subsets. In this paper only consider the situation of ongoing features. There are different actions [2], [8], [12] which can be applied to figure out how excellent a particular divided is for an attribute. Continuous 
feature divides are generally of the form attribute $1<\mathrm{X}$ or attribute1 $>$ X. Here have used C4.5 launch 8 in developing choice plants. Consider an ongoing feature $\mathrm{A}$ which requires on $\mathrm{N}$ exclusive principles (e.g. for $\mathrm{A}=3, \mathrm{~A}=5, \mathrm{~A}=7, \mathrm{~N}=3$ ). If the feature principles are categorized then there are $\mathrm{N}-1$ possible divided limits at $\mathrm{t}=\left(\mathrm{V}_{\mathrm{i}}+\mathrm{V}_{\mathrm{i}+1}\right) / 2$, where is a value of feature $A$ and $V_{i}<V_{j / i}<j$ so they are in categorized purchase. If one allows only binary divides then every threshold provides exclusive subsets 1 and 132 of the illustrations at node $\mathrm{K}$. The capability to select the limit to increase the breaking requirements prefers ongoing features with many exclusive principles. The threshold specification of parameter $\mathrm{K}$ is.

$$
\begin{aligned}
& \operatorname{Inf} o(K)=-\sum_{j=1}^{C} p(K, j) \times \log (p(Z K, j)) \\
& \operatorname{Gain}(G, T)=\inf o(K)-\sum_{i=1}^{L} \frac{K i}{K} \times \inf o(K i)
\end{aligned}
$$

The second part of developing an ultimate choice shrub is pruning the shrub to eliminate nodes that do not add accuracy and thereby decrease shrub dimension. Trimming is likely to be very important for huge coaching set which will generate large trees. There are a variety of techniques to trim a decision tree. In C4.5 a strategy known as pessimistic pruning is used. This strategy to pruning is very useful for little information places as it does not need a separate analyze set for the pruning procedure. Negative pruning is quite quick and has been proven to offer plants that perform effectively. However, it is compelled to use and calculate of mistake at any node in a choice shrub which is not clearly audio.

\section{BACKGROUND APPROACH}

No before perform concentrates on cross-domain privacypreserving inter firewall program marketing. This paper represents the first thing in discovering this unidentified area. Specifically, to concentrate on eliminating inter firewall program plan redundancies in a privacy-preserving way [4][7]. Consider two adjacent firewalls 1 and 2 that belong to different management domains and. Let signify the plan on firewall program 1's outgoing interface to firewall program 2 and signify the plan on firewall 2's inbound interface from firewall program 1. For a concept in, if all the packages that coordinate but do not coordinate any rule above in are eliminated by, concept can be removed because such packages never come to. Concept an inter firewall program repetitive concept with regard to. Observe that and only narrow the visitors from to; the visitors from firewall program 2's confident interface to firewall program 1's incoming interface are protected by other two individual guidelines. For convenience, believe that and have no intra firewall redundancy as such redundancy can be removed using the suggested alternatives.

The key task is to style a method that allows two adjacent fire walls to recognize the inter firewall program redundancy with regard to each other without understanding the plan of the other firewall program. While intra firewall program redundancy elimination is complex, inter firewall program redundancy elimination with the privacy-preserving need is even more complicated. To determine whether a concept in is inter firewall program repetitive with regard to , certainly needs some details about ; yet, cannot expose from such details.

An uncomplicated remedy is to execute a comfort preserving comparison between two guidelines from two nearby fire walls. Particularly, for each concept in, this remedy assessments whether all possible packages that coordinate concept in coordinate a rule with the eliminate choice in. If concept prevails, is inter-firewall redundant with regard to in. However, because firewalls adhere to the first-match semantics and the guidelines in a firewall typically overlap, this remedy is not only incorrect but also incomplete. Incorrect indicates that incorrect repetitive guidelines could be recognized in. Assume this remedy recognizes as a redundant rule in with regard to in. However, if some packages that coordinate concept also coordinate concept (is above) with the agree to choice in, these packages will pass through, and then needs to narrow them with. In this situation, is actually not repetitive. Imperfect indicates that a portion of repetitive guidelines could be recognized in. If all possible packages that coordinate concept in are eliminated by not only one concept but several guidelines in, is also repetitive. However, the immediate evaluation remedy cannot recognize such redundancies.

\section{PRIVACY PRESERVING FIREWALL CONFIGURATION}

First to coordinate the set of guidelines if it is identical guidelines it can be left out from any one of sector. That is the redundant concept can be removed from the different domain. $\mathrm{S}$ is the set of biggest guidelines. $\mathrm{i}$ and $\mathrm{j}$ are the different sector firewall program guidelines use the algorithm to coordinate set of guidelines is $\mathrm{M}(\mathrm{nr})$. Similar guidelines like a redundant concept are $\mathrm{R}$ (nr). If both are coordinate it removes the concept from any one of the firewall program and performs optimization.

This Method discovering inter-firewall repetitive rules in one firewall program with regard to another firewall program. To do this, first turn each firewall program to an equivalent sequence of no the actual guidelines.

- $\quad$ Produced from the two fire walls for detecting inter firewall redundancy.

- $\quad$ Recommend privacy-preserving

- Protocol for fixing each sub issue

The following criteria are used to discover biggest set of rules. That is very useful to decrease the repetitive concept. That is operating during a novel comfort used in execution of the project.

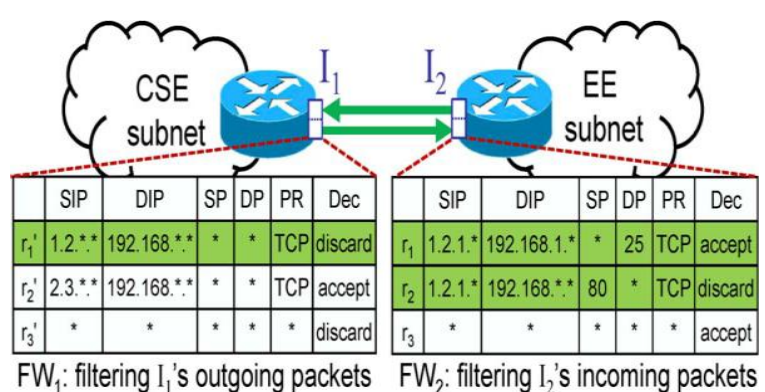

Fig 2: Cross domain specification for accessing firewalls 
In the following abstract sketch of a basic decision tree algorithm, all the steps described before are combined together:

1. Start with root node $\left(X_{t}=X\right)$.

2. Fpr each new node $t$.

- For each feature $x_{k}, k=1,2, \ldots, t$.

- For each threshold value $\alpha_{k \pi}, n=1,2, \ldots, N_{t k}$ -

* Generate $X_{t Y}$ and $X_{t N}$ according to the answer in the question: is $x_{k}$ (i) $\leq$ $\alpha_{\text {sin }}, i=1,2, \ldots, N_{t}$ -

* Compute the impurity decrease $(\Delta I(t))$.

- End.

- Choose oxro leading to the maximum with respect to $x_{k}$

- End

- Choose $x_{k_{0}}$ and associated $\alpha k_{0} n_{0}$ leading to the overall maximum decrease of impurity.

- If the stop-splitting rule is met, declare node $t$ as a leaf and designate it with a class label.

- If not, generate two descendant nodes $t_{y}$ and $t_{N}$ with associated subsets $X_{t Y}$ and $X_{t N}$, depending on the answer to the question: is $X_{k_{0}} \leq \alpha_{k_{0}} \leq \alpha_{k_{0} n_{0}}$.

3. End

Fig 3: Network address translation based on decision tree modulation

\section{EXPERIMENTAL EVALUATION}

Here analyzed the efficiency and efficiency of this protocol on 10 actual ACLs and 100 artificial ACLs. Both actual and synthetic ACLs analyze five areas, resource IP, location IP, source slot, location slot, and method kind. For actual ACLs, the variety of guidelines varies from countless numbers to countless numbers, and the regular variety of guidelines is 806. Due to protection issues, it is challenging to acquire many actual ACLs. For artificial ACLs, the variety of guidelines ranges from 200 to 2000, and for each variety, produced 10 synthetic ACLs. In applying the commutative protection, used the Pohlig-Hellman criteria with a 1024-bit prime modulus and 160-bit protection important factors. These experiments were applied using Coffee 1.6.0 and performed on a PC running a linux systemunix with 2 Apple Xeon cores and $16 \mathrm{~GB}$ of storage. To assess the efficiency, confirmed the correctness of this method because realized all the ACLs in the tests. The outcomes display that this method is deterministic and accurate with the given ACLs. Thus, in this area, to concentrate on the efficiency of this method. Remember that handling ACL Ai $(1 \leq i \leq n-1)$ is different from handling the last destination ACL An. Therefore to assess the calculations and communication costs of the primary functions of this method, processing ACL Ai $(1 \leq i \leq n-1)$, handling the location ACL An, and evaluating Ai and An. Understanding the efficiency of this protocol, you can calculate efforts and area consumption for a given system direction with $\mathrm{n}$ ACLs that belong to $\mathrm{n}$ events.

\subsection{Efficiency on Real ACLs}

This method is effective for handling actual ACL Ai $(1 \leq \mathrm{i} \leq$ $\mathrm{n}-1)$. Fig. 4 reveals for handling Ai the computation cost of $\mathrm{Pi}$ and the common calculations price of other parties $\mathrm{Pi}+1, \cdots$, $\mathrm{Pn}$. The calculations price of $\mathrm{Pi}$ is less than 2 seconds and the calculations price of $\operatorname{Pj}(i+1 \leq j \leq n)$ is less than 1.5 a few moments. Observe that, for handling $\mathrm{Ai}$, the computation price of $\mathrm{Pi}$ is one-time off-line price because $\mathrm{Pi}$ knows $\mathrm{Ai}$, while the calculations price of $\operatorname{Pj}(i+1 \leq j \leq n)$ is on the internet price. Fig. 4 reveals the common interaction cost between any two nearby events $\mathrm{Pj}$ and $\mathrm{Pj}+1(\mathrm{i} \leq \mathrm{j} \leq \mathrm{n})$ for handling ACL Ai, which is less than $60 \mathrm{~KB}$. Observe that, the calculations expenses of different events $P j(i+1 \leq j \leq n)$ are identical because they secure the same variety of prefixes from Ai. Hence only display the common calculations cost of events $\mathrm{Pi}+1, \cdots, \mathrm{Pn}$. In the same way, the interaction costs between every two nearby events $\mathrm{Pj}$ and $\mathrm{Pj}+1$ are the same.

\subsection{Efficiency on Synthetic ACLs}

The one-time offline computation price (i.e., the calculations price of Pn) is less than 550 a few moments, and the on the internet calculations price (i.e., the average computation price of other events $\mathrm{P} 1, \cdots, \mathrm{Pn}-1)$ is less than 25 a few moments.

The regular interaction price between $\mathrm{Pn}$ and $\mathrm{Pi}$ is less than $2100 \mathrm{~KB}$

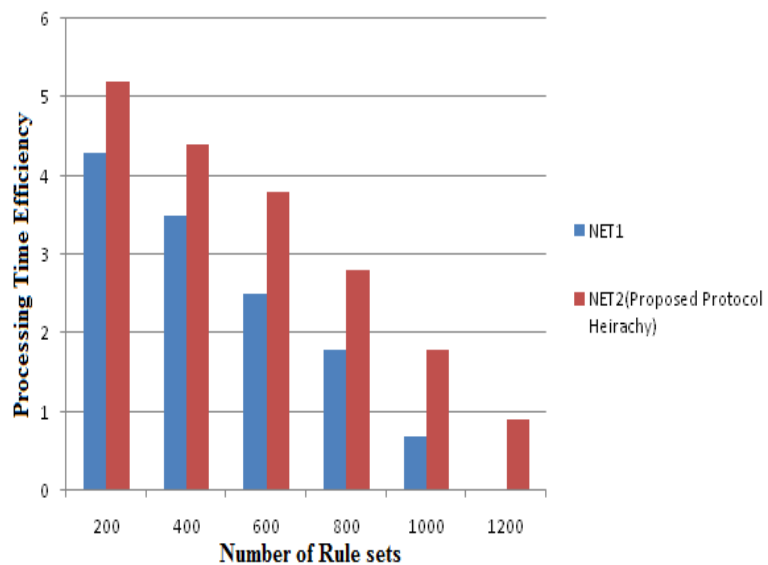

Fig 4: Processing time efficiency in real time firewall rule sets

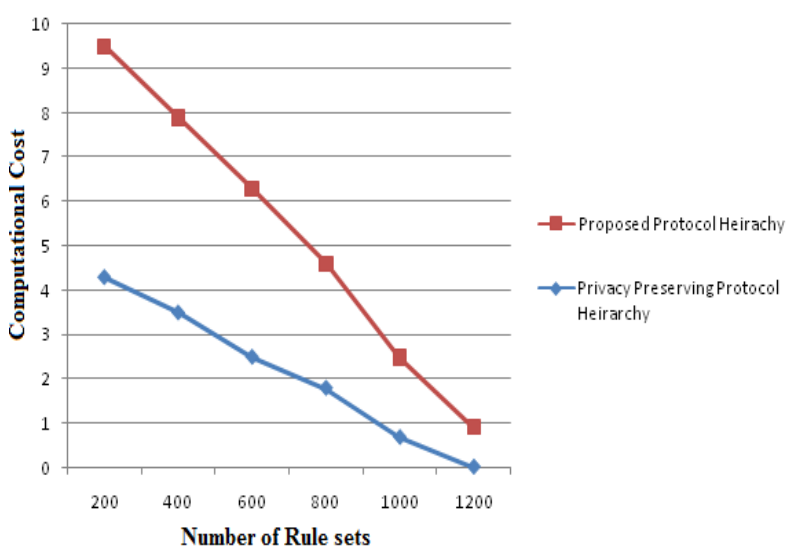

Fig 5: Computational overhead with firewall filtering rules 
This method accomplishes important redundancy rate on four real firewall program categories. For five actual firewall program categories, this protocol achieves a normal redundancy rate of $19.4 \%$. Particularly, for the firewall program team Variety, this method accomplishes $49.6 \%$ redundancy ratio, which indicates that almost 50 percent of guidelines in Host2 are inter-firewall repetitive guidelines. For firewall program categories this method accomplishes $14.4 \%-$ $17.1 \%$ redundancy percentages, which indicates that about $15 \%$ of guidelines in FW2 are redundant in these three categories. Only for one firewall program team, this method accomplishes $1.0 \%$ redundancy rate. From these outcomes, noticed that most nearby actual fire walls have many interfirewall redundant guidelines. Thus, this method could effectively remove inter-firewall repetitive guidelines and considerably improve the system efficiency.

Here this method is effective for handling and evaluating two real fire walls. When handling in the five actual firewall groups, the handling duration of is less than $2 \mathrm{sec}$ and the processing time of is less than $1 \mathrm{sec}$. When handling in those actual firewall program categories, the handling duration of is less than $4 \mathrm{sec}$ and the handling duration of is less than $10 \mathrm{sec}$. The comparison duration of two fire walls is less than 0.07 sec. The total processing duration of two events is less than 14 sec, which demonstrates the efficiency of this method. This method is effective for the interaction price between two events. When handling firewall program in the five actual firewall groups, the interaction price from to and that from to are less than $60 \mathrm{kB}$. Observe that the communication cost from to and that from to are the same because and secure the same number of principles and the secured principles have the same duration, i.e. 1024 pieces in our tests. When handling in those real firewall program categories, the interaction price from to is less than $300 \mathrm{kB}$. The complete interaction price between two parties is less than $350 \mathrm{kB}$, which can be sent through the current network (e.g. DSL network) around 10 sec.

The artificial firewalls also analyze the same five areas as actual fire walls. The number of guidelines in the artificial fire walls varies from 200 to 2000 , and for each variety produced 10 artificial fire walls. To evaluate the performance, first prepared each synthetic firewall as and then calculated the handling time and communication price of two events. Second prepared each synthetic firewall program as and calculated the handling time and interaction price. Third calculated the comparison time for every two artificial fire walls.

\section{CONCLUSION}

This approach resolved the issue of comfort preserving quantification of system reachability across different domains. Defending the comfort of accessibility management configuration is essential as the details can be quickly misused. This paper proposes an effective and protected method to evaluate the network reachability perfectly while protecting the comfort of ACLs. Here this system uses the divide-and-conquer way to break down the reachability calculations which outcomes in a scale reduction of the calculations and interaction expenses. To validate this method performed the tests on both actual and synthetic ACLs, which illustrate that this method has the benefits of quick calculations as well as low communication overhead, and is appropriate for use in implemented systems.

The upcoming perform will consider powerful redirecting information and topological modifications where hyperlinks go down or new links get included to the system leading to new routes for data propagation. This protocol is most beneficial if both parties are willing to benefit from it and can collaborate in a mutual manner. There are many special cases that could be explored based on this current protocol. For example, there may be hosts or Network Address Translation (NAT) devices between two adjacent firewalls. The current protocol cannot be directly applied to such cases. Extending this protocol to these cases could be an interesting topic and requires further investigation.

\section{REFERENCES}

[1] "Cross-Domain Privacy-Preserving Cooperative Firewall Optimization", by Fei Chen, in IEEE/ACM TRANSACTIONS ON NETWORKING, VOL. 21, NO. 3, JUNE 2013.

[2] nf-HiPAC, "Firewall throughput test," 2012 [Online]. Available: http://www.hipac.org/performance_tests/ results.html

[3] J. Brickell and V. Shmatikov, "Privacy-preserving graph algorithms in the semi-honest model," in Proc. ASIACRYPT, 2010, pp. 236-252.

[4] A. X. Liu and M. G. Gouda, "Complete redundancy removal for packet classifiers in TCAMs," IEEE Trans. Parallel Distrib. Syst., vol. 21, no. 4, pp. 424-437, Apr. 2010

[5] A. X. Liu, C. R. Meiners, and E. Torng, "TCAM Razor: A systematic approach towards minimizing packet classifiers in TCAMs," IEEE/ACM Trans. Netw., vol. 18, no. 2, pp. 490-500, Apr. 2010.

[6] A. X. Liu, C. R. Meiners, and Y. Zhou, "All-match based complete redundancy removal for packet classifiers in TCAMs," in Proc. IEEE INFOCOM, 2008, pp. 574-582.

[7] "Decision Tree Learning on Very Large Data Sets", by Lawrence O. Hall, Nitesh Chawla and Kevin W. Bowyer, in Proceedings of the 14th International Conference on Machine Learning, pp. 254-262, 1997.

[8] Y. Sang and H. Shen. Efficient and secure protocols for privacy-preserving set operations. ACM TISSEC, 13(9), 2009.

[9] S. Singh, F. Baboescu, G. Varghese, and J. Wang. Packet classification using multidimensional cutting. In SIGCOMM, 2003.

[10] Y.-W. E. Sung, C. Lund, M. Lyn, S. Rao, and S. Sen. Modeling and understanding end-to-end class of service policies in operational networks. In SIGCOMM, 2009.

[11] A. Wool. A quantitative study of firewall configuration errors. IEEE Computer, 37(6), 2004.

[12] A. X. Liu and M. G. Gouda, "Complete redundancy removal for packet classifiers in TCAMs," IEEE Trans. Parallel Distrib. Syst., vol. 21, no. 4, pp. 424-437, Apr. 2010.

[13] Q. Dong, S. Banerjee, J. Wang, D. Agrawal, and A. Shukla, "Packet classifiers in ternary CAMs can be smaller," in Proc. ACM SIGMETRICS.

[14] E. Al-Shaer and H. Hamed, "Discovery of policy anomalies in distributed firewalls," in Proc. IEEE INFOCOM, 2004. 